Pacific Journal of Mathematics

ALGEBRAIC AUTOMORPHISMS OF ALGEBRAIC GROUPS 


\title{
ALGEBRAIC AUTOMORPHISMS OF ALGEBRAIC GROUPS WITH STABLE MAXIMAL TORI
}

\author{
SARAH J. GotTLieb
}

\begin{abstract}
Let $T_{1}$ and $T_{2}$ be maximal tori of a connected linear algebraic group $G \subseteq G L(n, \kappa)$, and suppose some (algebraic group) automorphism $\sigma$ of $G$ stabilizes both $T_{1}$ and $T_{2}$. Suppose further that $\sigma$ also stabilizes two Borel subgroups, $B_{1}$ and $B_{2}$, of $G$. This paper is about the following natural questions:
\end{abstract}

(1) Are $T_{1}$ and $T_{2}$ conjugate by a $\sigma$-fixed point of $G$ ?

(2) Are $B_{1}$ and $B_{2}$ conjugate by a $\sigma$-fixed point of $G$ ?

(3) If $T_{i} \subseteq B_{i},(i=1,2)$, are the $T_{i}$ and $B_{i}$ respectively conjugate by a single $\sigma$-fixed point of $G$ ?

(4) Are at least $T_{1}$ and $T_{2}$ described in (3) above conjugate by a $\sigma$-fixed point of $G$ ?

In this paper is treated the case in which $\sigma$ is an algebraic automorphism. If either $p=$ char $\kappa=0$ or $\sigma$ is semisimple, then the answer to (4) above is yes; but there are counterexamples for (1), (2), and (3). (See below, Counterexamples A-1 and B.) Moreover, if both $p>0$ and $\sigma$ is not semisimple, then there is also a counterexample for question (4). (See below, Counterexample C.)

Incidental in the proofs is the simple result that when $\sigma$ is algebraic, a $\sigma$-stable maximal torus is pointwise fixed by some finite power of $\sigma$, and by $\sigma$ itself for $p=0, \sigma$ unipotent (Theorem 1).

Robert Steinberg has studied the questions above in [3], for the case that $\sigma$ has finite fixed-point set in $G$, finding that the answers to questions (2), (3), and (4) are all yes. There is a counterexample for question (1) in the finite fixed-point set case, when the $\sigma$-stable maximal tori are not respectively contained in $\sigma$-stable Borel subgroups. (See below, Counterexample A-2.)

When $\sigma$ is an algebraic automorphism of a general algebraic group $G$, its fixed-point set may be infinite. In fact, Steinberg shows (by [3], 10.10) that if $\sigma$ is algebraic with finite fixed-point set, then $G$ is necessarily solvable.

Throughout the paper the (now standard) terminology and basic results of Borel ([1] and [2]) are used, including the name Borel subgroup for a maximal solvable connected subgroup. In addition the mnemonic clag is used for a connected linear algebraic group, and the expression "the pair $T \subseteq B$ " for a maximal torus $T$ and a Borel subgroup $B$ containing $T$.

In all of the following theorems, $G$ is a clag and $\sigma$ an algebraic automorphism of $G$. 
THEOREM 1. If $G$ has a $\sigma$-stable maximal torus $T$, then $T$ is pointwise-fixed by some power $\sigma^{n}$ of $\sigma$. If $p=0$ and $\sigma$ is unipotent, then $T$ is pointwise fixed by $\sigma$.

Proof. Since $\sigma$ is an algebraic automorphism of $G$, there is a closed linear algebraic group $\mathscr{G}$ with $G \Delta \mathscr{G}$ and an element $s \in \mathscr{G}$ such that $\sigma(g)=s g s^{-1}$ for each $g \in G$. (In fact this may be taken as the definition for an algebraic automorphism of $G$.)

Form the algebraic group generated by $T$ and $s, \mathscr{A}(T, s)=\mathscr{A}$ in $\mathscr{G}$ (see [1], $\S 3$ ). $T$ is normalized by $s$, so $T$ is normal in $\mathscr{A}$. Moreover, $T$ is a torus in $\mathscr{A}_{0}$, and so is contained in a maximal torus of $\mathscr{A}_{0}$. Thus $T$ is contained in every maximal torus of $\mathscr{A}_{0}$, hence is contained centrally in every Borel subgroup of $\mathscr{A}_{0}$ by ([1], $\S 18,18.1)$. $T$ is therefore central in $\mathscr{A}_{0}$ by $([1], \S 18,18.5)$.

Now $s \in \mathscr{A} \Rightarrow$ some power $s^{n}$ of $s$ is in $\mathscr{A}_{0}$, whence $s^{n}$ centralizes $T$. Equivalently, $\sigma^{n}$ fixes $T$ pointwise.

Suppose now that $p=0$ and $\sigma$ is unipotent. Since $s^{n}$ centralizes $T$; so does $\mathscr{A}\left(s^{n}\right)$. Now $\sigma$ unipotent $\Rightarrow s$ unipotent; and for $p=0$, $\mathscr{A}\left(s^{n}\right)=\mathscr{A}(s)$ (see [1], 8.2). Thus $s$ also centralizes $T$, i.e., $\sigma$ fixes $T$ pointwise.

Theorem 2. Let $G$ be solvable, and let either $p=0$ or $\sigma$ be semisimple. Then two $\sigma$-stable maximal tori $T_{i}$ of $G(i=1,2)$ are conjugate by a $\sigma$-fixed point of $G$.

Proof. (1) Since $\sigma$ has finite order, say $n$ ( $n$ is prime to $p$ when $p>0$ ), on $T_{1}$ and $T_{2}$, it may be assumed without change in hypothesis that $\sigma$ has such finite order on all of $G$, by replacing $G$ with $\left(G_{\sigma^{n}}\right)_{0}$, the connected component of the set of $\sigma^{n}$-fixed points in G.

(2) Let $U$ be the unipotent part of $G$, and let $V$ be the unipotent part of $C\left(T_{1}\right)$, the Cartan subgroup of $T_{1}$. There exists $u \in U$ such that $u T_{1} u^{-1}=T_{2}$, and for any such $u, u^{-1} \cdot \sigma(u) \in V$. Therefore it suffices to show that whenever there is a $u$ with $u^{-1} \cdot \sigma(u) \in V$, then there must exist $v \in V$ with $u^{-1} \cdot \sigma(u)=v^{-1} \cdot \sigma(v)$. For in that case, $u v^{-1}$ is $\sigma$-fixed with $u v^{-1} T_{1} v u^{-1}=T_{2}$.

In view of (1) and (2) it suffices to prove the following lemma:

LEMMA 3. Let $G$ be a unipotent clag with automorphism $\sigma$ of finite order $n$ (prime to $p$ when $p>0$ ). If $G$ has a $\sigma$-stable subclag $H$, and an element $g \in G$ such that $g^{-1} \cdot \sigma(g) \in H$, then $\exists h \in H$ such that $g^{-1} \cdot \sigma(g)=h^{-1} \cdot \sigma(h)$.

Proof. For any subset $X$ of $G$, denote by $X_{\sigma}$ the $\sigma$-fixed point 
set of $X$; and for any element $x \in G$, set $\alpha(x)=x^{-1} \cdot \sigma(x)$. There is no non-identity element of the form $\alpha(x)$ in $G_{o}$, because if $\alpha(x) \in G_{\sigma}$ for some $x \in G$, then

$$
\begin{aligned}
(\alpha(x))^{n} & =\alpha(x) \cdot \sigma(\alpha(x)) \cdot \sigma^{2}(\alpha(x)) \cdots \sigma^{n-1}(\alpha(x)) \\
& =x^{-1} \cdot \sigma(x) \cdot \sigma\left(x^{-1}\right) \cdot \sigma^{2}(x) \sigma^{2}\left(x^{-1}\right) \cdots \sigma^{n-1}\left(x^{-1}\right) \sigma^{n}(x) \\
& =x^{-1} \sigma^{n}(x)=e
\end{aligned}
$$

but only the identity element can be both unipotent and of order $n$.

Case I. $H$ normal in $G$. $H$ is unipotent, hence nilpotent, so one may use induction on the length $l$ of the lower central series for $H$.

If $l=1$, then $H$ is commutative, so $\left.\alpha\right|_{H}$ is an endomorphism of $H$ with kernel $H_{\sigma}$ and image $\alpha(H)$. Therefore $\operatorname{dim} H=\operatorname{dim} H_{0}+$ $\operatorname{dim} \alpha(H)$, and $H_{\sigma} \cap \alpha(H)=\{e\}$. So $H=H_{\sigma} \cdot \alpha(H)$ as a direct product.

Thus $\exists h_{1} \in H_{\sigma}, h_{2} \in H$ such that $\alpha(g)=h_{1} \cdot \alpha\left(h_{2}\right)$. That is, $g^{-1} \cdot \sigma(g)=$ $h_{1} \cdot h_{2}^{-1} \cdot \sigma\left(h_{2}\right)=h_{2}^{-1} \cdot h_{1} \cdot \sigma\left(h_{2}\right)$; and this implies that

$$
h_{2} \cdot g^{-1} \cdot \sigma(g) \sigma\left(h_{2}^{-1}\right)=\left(g h_{2}^{-1}\right)^{-1} \cdot \sigma\left(g h_{2}^{-1}\right)=\alpha\left(g h_{2}^{-1}\right)=h_{1} \in H_{\sigma} \leqq G_{\sigma} .
$$

So $\alpha\left(g h_{2}^{-1}\right)=e=h_{1}$ and $\alpha(g)=\alpha\left(h_{2}\right)$.

Now suppose $l>1$. If $\alpha(g) \in H^{1}$, then by induction $\exists h \in H^{1}$ with $\alpha(g)=\alpha(h)$. So suppose $\alpha(g) \notin H^{1}$. Then $\overline{\alpha(g)} \neq \bar{e}$ in $\bar{H}=\pi_{H^{1}}(H)$, where $\pi_{H^{1}}$ is the projection of $G$ with kernel $H^{1} \cdot \bar{H}$ is commutative, and $\overline{\alpha(g)}=\overline{g^{-1} \cdot \sigma(g)}=\bar{g}^{-1} \cdot \bar{\sigma}(\bar{g})=\bar{\alpha}(\bar{g})$, so as in the case for $l=1$, $\exists \bar{h} \in \bar{H}$ such that $\overline{\alpha(g)}=\overline{\alpha(h)}$. That is,

$$
\bar{g}^{-1} \cdot \bar{\sigma}(\bar{g})=\bar{h}^{-1} \cdot \bar{\sigma}(\bar{h}) \text {, and } \overline{\left(g h^{-1}\right)^{-1} \cdot \sigma\left(g h^{-1}\right)}=\bar{e} .
$$

In other words, $\alpha\left(g h^{-1}\right) \in H^{1}$, whence by induction $\exists h^{\prime} \in H^{1}$ such that $\alpha\left(g h^{-1}\right)=\alpha\left(h^{\prime}\right)$. We now have $\left(g h^{-1}\right)^{-1} \sigma\left(g h^{-1}\right)=h g^{-1} \sigma(g) \sigma(h)^{-1}=$ $h^{\prime-1} \cdot \sigma\left(h^{\prime}\right)$, implying $g^{-1} \cdot \sigma(g)=h^{-1} h^{\prime-1} \cdot \sigma\left(h^{\prime}\right) \sigma(h)=\left(h^{\prime} h\right)^{-1} \sigma\left(h^{\prime} h\right)$. Hence $\alpha(g)=\alpha\left(h^{\prime} h\right) \in \alpha(H)$.

Case II. If $H$ is not normal in $G$, set $H=G_{1}$, and let $G_{i}$ be the connected normalizer in $G$ of $G_{i-1}$, for $i \geqq 2$. Since a proper subclag of a nilpotent clag is properly contained in its connected normalizer by ([1], 20.3), there is a chain of $\sigma$-stable subclags of $G$ :

$$
H=G_{1} \underset{+}{\Delta} G_{2} \underset{+}{\Delta} \cdots \underset{+}{\Delta} G_{r}=G,
$$

each of which is a normal and proper subclag of the following one.

Now the element $g \in G$ with which we are concerned is contained in $G_{i}$ for some (minimal) $i$, with $i \geqq 2$. Since $\alpha(g) \in H \subseteq G_{i-1}$, and $G_{i-1}$ 
is normal in $G_{i}$, there is by Case I an element $g_{i-1} \in G_{i-1}$ for which $\alpha(g)=\alpha\left(g_{i-1}\right)$.

If $(i-1) \geqq 2$, apply Case I again to obtain an element $g_{i-2} \in G_{i-2}$ for which $\alpha\left(g_{i-1}\right)=\alpha\left(g_{i-2}\right)$, since $\alpha\left(g_{i-1}\right) \in H \leqq G_{i-2}$, and $G_{i-2}$ is normal in $G_{i-1}$.

Similarly, by a total of $(i-1)$ application of Case I, one obtains an element $h \in H=G_{1}=G_{i-(i-1)}$, for which $\alpha(h)=\alpha\left(g_{2}\right)=\alpha\left(g_{3}\right)=\cdots=$ $\alpha\left(g_{i-1}\right)=\alpha(g)$.

This completes the proof of Theorem 2 .

Theorem 4. Let $G$ have two $\sigma$-stable pairs, $T_{i} \leqq B_{i}(i=1,2)$. If $p=0$, or if $\sigma$ is semisimple, then the $T_{i}(i=1,2)$ are conjugate by a $\sigma$-fixed point of $G$.

Proof. Let $T \subseteq B$ be any $\sigma$-stable pair of $G$.

First consider $\sigma_{s}$, the semisimple component of $\sigma$. (Any $\sigma$-stable clag is also $\sigma_{s}$-stable.)

Let $S$ be a maximal torus of $\left(G_{\sigma_{s}}\right)_{0}$. By ([3], 7.4), $S \cong a \sigma_{s}$-stable Borel subgroup $R$ of $G$. $S$ is also a maximal torus of $\left(R_{\sigma_{s}}\right)_{0}$.

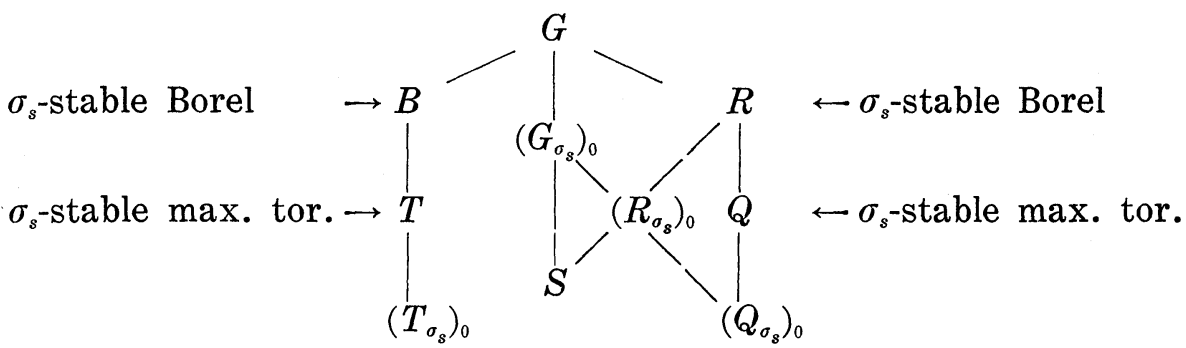

By ([3], 7.6), $R$ has a $\sigma_{s}$-stable maximal torus $Q$. Now $R=Q \cdot V$ (semi-direct product), where $V$ is the unipotent part of $R$. So any $\sigma_{s}$-fixed point $f \in R$ has Jordan decomposition $f=q \cdot v$ for some $q \in Q$, $v \in V$. Thus $f=\sigma_{s}(f)=\sigma_{s}(q) \sigma_{s}(v)$, with $\sigma_{s}(q) \in Q, \sigma_{s}(v) \in V$, whence $\sigma_{s}(q)=q$ and $\sigma_{s}(v)=v$. Hence $\left(R_{\sigma_{s}}\right)_{0}=\left(Q_{\sigma_{s}}\right)_{0} \cdot\left(V_{\sigma_{s}}\right)_{0}$, and $\left(Q_{\sigma_{s}}\right)_{0}$ is a maximal torus of $\left(R_{\sigma_{s}}\right)_{0}$. Thus $\operatorname{dim}\left(Q_{\sigma_{s}}\right)_{0}=\operatorname{dim} S$, so $\left(Q_{\sigma_{s}}\right)_{0}$ is also a maximal torus of $\left(G_{\sigma_{s}}\right)_{0}$.

Now $\exists g \in G$ such that $g R g^{-1}=B, g Q g^{-1}=T$, and (since $Q \subseteq R$, $T \subseteq B$ are all $\sigma_{s}$-stable), $g^{-1} \cdot \sigma_{s}(g) \in N_{G}(R) \cap N_{G}(Q)=R \cap N_{G}(Q)=C(Q)$, the Cartan subgroup of $Q$ in $G$. This implies that $g\left(Q_{\sigma_{s}}\right)_{0} g^{-1}=\left(T_{\sigma_{s}}\right)_{0}$, so that $\operatorname{dim}\left(T_{\sigma_{s}}\right)_{0}=\operatorname{dim}\left(Q_{\sigma_{s}}\right)_{0}$, and $\left(T_{\sigma_{s}}\right)_{0}$ is itself a maximal torus of $\left(G_{\sigma_{s}}\right)_{0}$.

Moreover, $\left(T_{\sigma_{s}}\right)_{0}$ is a torus of $\left(G_{\sigma_{u}}\right)_{0}$, because $T \cong\left(G_{\sigma_{u}}\right)_{0}$. Therefore $\left(T_{\sigma_{s}}\right)_{0}$ is a maximal torus of $\left(G_{\sigma_{s}}\right)_{0} \cap\left(G_{\sigma_{u}}\right)_{0}=\left(G_{\sigma}\right)_{0}$. Thus the $\left[\left(T_{i}\right)_{o_{s}}\right]_{0}$ are both maximal tori of $\left(G_{\sigma}\right)_{0}$; so they are conjugate by a fixed point $y \in\left(G_{\sigma}\right)_{0}$, that is, $y\left(T_{1_{\sigma_{s}}}\right)_{0} y^{-1}=\left(T_{2_{\sigma_{s}}}\right)_{0}$. Set $T_{3}=y T_{1} y^{-1}$. 
Both $T_{2}$ and $T_{3}$ belong to the connected centralizer $Z$ of $\left(T_{2_{\sigma_{s}}}\right)_{0}$ in $G$. By ([4], Cor. 4), $Z$ is solvable. Also, $Z$ is $\sigma$-stable with maximal tori $T_{2}$ and $T_{3}$, so by (Thm. 2), $T_{2}$ and $T_{3}$ are conjugate under a $\sigma$ fixed point $z \in Z$; that is, $z T_{2} z^{-1}=T_{3}$. Then for $g=y^{-1} z, g$ is a $\sigma$ fixed point of $G$ for which $g T_{2} g^{-1}=T_{1}$.

[Note on the field of definition $\kappa$ : If $\kappa$ is algebraically closed, the point of conjugacy in Theorems 2 and 4 may be taken to be $\kappa$-rational; and theorems analogous to Theorems 2 and 4 hold for $\kappa$-groups. The proofs are mechanical glosses on those here and are found in the author's $\mathrm{Ph}$. D. thesis.]

Counterexample A-1. $\sigma$ is semisimple; $G$ has two $\sigma$-stable maximal tori which are not both contained in $\sigma$-stable Borel subgroups, and are not conjugate by a $\sigma$-fixed point:

Take $G=S L(2, \Omega), p \neq 2$. Let $T_{1}$ consist of matrices of the form

$$
\left[\begin{array}{cc}
\alpha & 0 \\
0 & \frac{1}{\alpha}
\end{array}\right], \quad \alpha \neq 0 ;
$$

and let $T_{2}$ be given by matrices of the form

$$
\left[\begin{array}{ll}
\frac{1}{2}\left(\alpha+\frac{1}{\alpha}\right), & \frac{1}{2}\left(\alpha-\frac{1}{\alpha}\right) \\
\frac{1}{2}\left(\alpha-\frac{1}{\alpha}\right), & \frac{1}{2}\left(\alpha+\frac{1}{\alpha}\right)
\end{array}\right], \quad \alpha \neq 0 .
$$

( $T_{1}$ is the maximal torus of $G$ which has diagonal form; $T_{2}$ is the conjugate of $T_{1}$ by the element

$$
\frac{\sqrt{2}}{2}\left[\begin{array}{rr}
1 & 1 \\
-1 & 1
\end{array}\right] \in G
$$

Take $\sigma=\operatorname{Inn}_{G} g$, where $g=\left[\begin{array}{ll}0 & 1 \\ 1 & 0\end{array}\right]$. The effect of $\sigma$ is to interchange diagonally the corner entries in each matrix of $G$. The $\sigma$ fixed point set $G_{\sigma}$ of $G$ is therefore

$$
G_{\sigma}=\left\{\left[\begin{array}{ll}
a & b \\
b & a
\end{array}\right]: a^{2}-b^{2}=1\right\} \text {. }
$$

$G_{\sigma}$ is infinite; and since $\sigma^{2}=1$ and $p \neq 2, \sigma$ is semisimple.

Now $T_{2}$ is pointwise $\sigma$-fixed, and $T_{1}$ is not, although it is $\sigma$ stable. So $T_{1}$ and $T_{2}$ cannot be conjugate by a $\sigma$-fixed point of $G$. (Note. The only Borel subgroups of $G$ containing $T_{1}$ are the 
upper and lower triangular matrix groups in $G$, and $\sigma$ leaves neither of these stable, but maps one onto the other.)

Counterexample A-2. $\sigma$ (nonalgebraic) is the Frobenius map for $p=2$, having finite fixed-point set; $G$ has two $\sigma$-stable maximal tori which are not both contained in a $\sigma$-stable Borel subgroup, and are not conjugate by a $\sigma$-fixed point.

Take $G=S L(2, \Omega), p=2$. Let

$$
T_{1}=\left\{\left[\begin{array}{cc}
\alpha & 0 \\
0 & \frac{1}{\alpha}
\end{array}\right]: 0 \neq \alpha \in \Omega\right\} ;
$$

and let

$$
\left.\begin{array}{r}
T_{2}=\left\{\begin{array}{r}
\alpha+a\left(\alpha+\frac{1}{\alpha}\right),\left(\alpha+\frac{1}{\alpha}\right) \\
\left(\alpha+\frac{1}{\alpha}\right), \frac{1}{\alpha}+\alpha\left(\alpha+\frac{1}{\alpha}\right)
\end{array}\right]: 0 \neq \alpha \in \Omega ; \\
a \text { fixed such that } a^{2}+a+1=0
\end{array}\right\}
$$

For $\sigma$ take the Frobenius map $\sigma:\left(x_{i j}\right) \rightarrow\left(x_{i j}^{2}\right) . \quad T_{1}$ is clearly $\sigma$ stable. $T_{2}=x T_{1} x^{-1}$, where

$$
x=\left[\begin{array}{cc}
a & (a+1) \\
(a+1) & a
\end{array}\right], \text { and } x^{-1} \cdot \sigma(x)=\left[\begin{array}{ll}
0 & 1 \\
1 & 0
\end{array}\right] \in N\left(T_{1}\right),
$$

so $T_{2}$ is $\sigma$-stable too.

It can easily be seen that $T_{1}$ and $T_{2}$ are not conjugate by a $\sigma$ fixed point of $G$, since there are only 6 fixed points.

Counterexample B. $\sigma$ is semisimple; $G$ has two $\sigma$-stable pairs; but the $\sigma$-stable Borel subgroups are not conjugate by a $\sigma$-fixed point.

Take $G$ and $\sigma$ as in Counterexample A-1 $(p \neq 2)$. Let

$$
T=T_{2}=\left\{\left[\begin{array}{ll}
\frac{1}{2}\left(\alpha+\frac{1}{\alpha}\right) & \frac{1}{2}\left(\alpha-\frac{1}{\alpha}\right) \\
\frac{1}{2}\left(\alpha-\frac{1}{\alpha}\right) & \frac{1}{2}\left(\alpha+\frac{1}{\alpha}\right)
\end{array}\right]: 0 \neq \alpha \in \Omega\right.
$$

Set $\Delta=\left\{\left[\begin{array}{cr}\alpha & \beta \\ 0 & 1 / \alpha\end{array}\right]: \alpha, \beta \in \Omega, \alpha \neq 0\right\} \subseteq G$, a Borel subgroup of $G$. Set 


$$
x=\left[\begin{array}{rr}
1 & -\frac{1}{2} \\
1 & \frac{1}{2}
\end{array}\right] \in G, \text { and } y=\left[\begin{array}{rr}
1 & \frac{1}{2} \\
-1 & \frac{1}{2}
\end{array}\right] \in G
$$

Take

$$
B_{1}=x \Delta x^{-1}=\left\{\left[\begin{array}{l}
\frac{1}{2}\left(\alpha+\frac{1}{\alpha}\right)-\beta, \frac{1}{2}\left(\alpha-\frac{1}{\alpha}\right)+\beta \\
\frac{1}{2}\left(\alpha-\frac{1}{\alpha}\right)-\beta, \frac{1}{2}\left(\alpha+\frac{1}{\alpha}\right)+\beta
\end{array}\right]: \alpha, \beta \in \Omega, \alpha \neq 0\right\}
$$

and

$$
B_{2}=y \Delta y^{-1}=\left\{\left[\begin{array}{l}
\frac{1}{2}\left(\frac{1}{\alpha}+\alpha\right)+\beta, \frac{1}{2}\left(\frac{1}{\alpha}-\alpha\right)+\beta \\
\frac{1}{2}\left(\frac{1}{\alpha}-\alpha\right)-\beta, \frac{1}{2}\left(\frac{1}{\alpha}+\alpha\right)-\beta
\end{array}\right]: \alpha, \beta \in \Omega, \alpha \neq=0\right\} \text {. }
$$

Recalling that $\sigma$ diagonally interchanges the entries of a matrix, one sees that $B_{1}$ and $B_{2}$ are $\sigma$-stable, and $T$ is pointwise $\sigma$-fixed. Moreover, $T$ is clearly a maximal torus of both $B_{1}$ and $B_{2}$ (i.e., when $\beta=0$ ). So $T \varsubsetneqq B_{1}$ and $T \varsubsetneqq B_{2}$ are $\sigma$-stable pairs.

Suppose now that $B_{1}, B_{2}$ are conjugate by a $\sigma$-fixed point $f \in G_{\sigma}$, i.e., that $B_{1}=f B_{2} f^{-1}$. Then $B_{1}=x \Delta x^{-1}=f B_{2} f^{-1}=f y \Delta y^{-1} f^{-1} \Rightarrow \Delta=$ $x^{-1} f y \Delta y^{-1} f^{-1} x \Rightarrow x^{-1} f y \in N_{G}(\Delta)=\Delta$.

Say that $x^{-1} f y=b=\left[\begin{array}{rr}\alpha & \beta \\ 0 & 1 / \alpha\end{array}\right] \in \Delta$, and $f=\left[\begin{array}{ll}\gamma & \delta \\ \delta & \gamma\end{array}\right] \in G_{o}$, for some $\alpha, \beta, \gamma, \delta \in \Omega$ with $\gamma^{2}-\delta^{2}=1$, and $\alpha \neq 0$. Then

$$
\begin{aligned}
x^{-1} f y=b & \Longrightarrow f y=x b \\
& \Longrightarrow\left[\begin{array}{ll}
\gamma & \delta \\
\delta & \gamma
\end{array}\right]\left[\begin{array}{rr}
1 & \frac{1}{2} \\
-1 & \frac{1}{2}
\end{array}\right]=\left[\begin{array}{rr}
1 & -\frac{1}{2} \\
1 & \frac{1}{2}
\end{array}\right]\left[\begin{array}{ll}
\alpha & \beta \\
0 & \frac{1}{\alpha}
\end{array}\right] \\
& \Longrightarrow\left[\begin{array}{ll}
\gamma-\delta & \frac{1}{2}(\gamma+\delta) \\
\delta-\gamma & \frac{1}{2}(\gamma+\delta)
\end{array}\right]=\left[\begin{array}{ll}
\alpha & \beta-\frac{1}{2 \alpha} \\
\alpha & \beta+\frac{1}{2 \alpha}
\end{array}\right] \\
& \Longrightarrow \gamma-\delta=\delta-\gamma \Rightarrow \gamma=\delta \Rightarrow \gamma^{2}-\delta^{2}=0,
\end{aligned}
$$

a contradiction of the fact that $\gamma^{2}-\delta^{2}=1$.

Thus $B_{1}$ and $B_{2}$ cannot be conjugate by a $\sigma$-fixed point of $G$.

Counterexample C. $G$ solvable, $\sigma$ unipotent, and $p>0 . G$ has 
two $\sigma$-stable maximal tori which are not conjugate by a $\sigma$-fixed point.

Take $p=2$.

Let $T$ be the torus $\subseteq G L(6, \Omega)$ consisting of diagonal matrices $t$ of the form

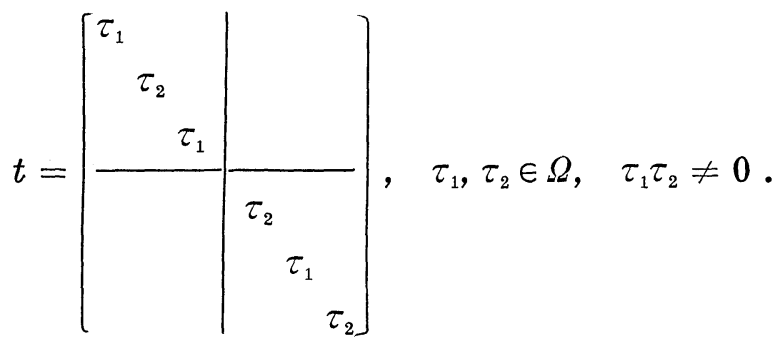

Let $U$ be the unipotent clag consisting of upper triangular matrices $u$ of the form

$$
u=\left[\begin{array}{lll|lll}
1 & \alpha & x & & & \\
& 1 & \beta & & & \\
& & 1 & & & \\
\hline & & & 1 & \beta & y \\
& & & 1 & \alpha \\
& & & & & 1
\end{array}\right], \quad \alpha, \beta, x, y \in \Omega,
$$

satisfying: $x+y-\alpha \beta=0$.

The reader may verify that $U$ is closed under multiplication, and since $u^{4}=e, \forall u \in U, U$ is also closed under inverses. Hence $U$ is well-defined.

Moreover, $U$ is normalized by $T$, as the reader again may verify.

One may therefore form the solvable clag $G=T \cdot U$ (semi-direct product).

Let the automorphism $\sigma$ on $t \cdot u \in G$ be given by the following action on the entries of $t$ and $u$

$$
\begin{aligned}
\sigma: \tau_{1} & \longleftrightarrow \tau_{2} \\
\alpha & \longleftrightarrow \beta \\
x & \longleftrightarrow y
\end{aligned}
$$

$\sigma$ is thus conjugation by the permutation matrix:

$$
s=\left[\begin{array}{lll|lll} 
& & & 1 & & 0 \\
& 0 & & & 1 & \\
\hline 1 & & 0 & & & \\
& 1 & & & 0 & \\
0 & & 1 & & &
\end{array}\right] .
$$


So $s$ and $\sigma$ are unipotent of order 2 .

$T$ is a $\sigma$-stable maximal torus of $G$, whose Cartan subgroup is $C(T)=T \times C(T)_{u}$, where

$$
C(T)_{u}=\left\{\left(\left[\begin{array}{ccc|ccc}
1 & 0 & x & & & \\
& 1 & 0 & & 0 & \\
0 & & 1 & & & \\
\hline & & 1 & 0 & x \\
& 0 & & & 1 & 0 \\
& & & 0 & & 1
\end{array}\right]: x \in \Omega\right\} .\right.
$$

Now if $u \in U$, then $u T u^{-1}$ is $\sigma$-stable if and only if $u^{-1} \cdot \sigma(u) \in C(T)_{u}$. Moreover, $\exists$ a $\sigma$-fixed element $f \in U_{o}$ such that $u T u^{-1}=f T f^{-1}$ if and only if $f^{-1} u \in C(T)_{u}$; i.e., if and only if $\exists c \in C(T)_{u}$ such that $u c^{-1}=f$ is $\sigma$-fixed.

However, all $c \in C(T)_{u}$ are $\sigma$-fixed; So a $\sigma$-stable maximal torus $u T u^{-1}$ of $G$ is conjugate to $T$ by a $\sigma$-fixed point if and only if $u$ itself is $\sigma$-fixed.

However, for the unipotent matrix

$$
u=\left[\begin{array}{lll|lll}
1 & \alpha & x & & & \\
& 1 & \alpha & & 0 & \\
0 & & 1 & & & \\
\hline & & & 1 & \alpha & y \\
& 0 & & & 1 & \alpha \\
& & & 0 & & 1
\end{array}\right]
$$

satisfying $x+y-\alpha^{2}=0, \alpha \neq 0$, one gets

$$
u^{-1} \cdot \sigma(u)=\left[\begin{array}{ccc|ccc}
1 & 0 & -x+y & & & \\
& 1 & 0 & & 0 & \\
0 & & 1 & & & \\
\hline & & & 1 & 0 & -x+y \\
& 0 & & & 1 & 0 \\
& & & 0 & & 1
\end{array}\right] .
$$

That is, $u^{-1} \cdot \sigma(u) \in C(T)_{u}$, so $u T u^{-1}$ is $\sigma$-stable. But $u$ is not $\sigma$-fixed, so $T$ and $u T u^{-1}$ are not conjugate by a $\sigma$-fixed element of $G$.

(Note. This counterexample in $p=2$ is due to D. Winter. The present author has generalized it in a separate paper for all $p>0$. The resulting group may be of some interest in itself.) 


\section{REFERENCES}

1. Armand Borel, Groupes lineares algebriques, Annals of Math., 64 (1956), 20-82.

2. — Linear Algebraic Groups, W. A. Benjamin, New York, 1969.

3. Robert Steinberg, Endomorphisms of linear algebraic groups, A. M. S. Memoirs, 80 (1968).

4. David Winter, On automorphisms of algebraic groups, Bull. A. M. S., 72 (1966), 706-708.

5. — Algebraic group automorphisms having finite-fixed point sets, preprint, 1966.

Received January 12, 1977 and in revised form March 23, 1977. This work represents part of the author's doctoral dissertation Rutgers University, May, 1973. The author wishes to express her gratitude to her advisor, Professor Earl Taft, for his guidance and encouragement.

PURdUE UNIVERSITY

WeSt LAFAYETTE, IN 47907 


\title{
PACIFIC JOURNAL OF MATHEMATICS
}

\section{EDITORS}

\author{
RICHARD ARENS (Managing Editor) \\ University of California \\ Los Angeles, CA 90024 \\ Charles W. Curtis \\ University of Oregon \\ Eugene, OR 97403 \\ C. C. MOORE \\ University of California \\ Berkeley, CA 94720
}

\section{J. DUGUNDJI}

Department of Mathematics

University of Southern California

Los Angeles, CA 90007

R. FINN and J. MILGRAM

Stanford University

Stanford, CA 94305

\section{ASSOCIATE EDITORS}
E. F. BECKENBACH
B. H. NeumanN
F. WOLF
K. YoSHIDA

\section{SUPPORTING INSTITUTIONS}

UNIVERSITY OF BRITISH COLUMBIA

CALIFORNIA INSTITUTE OF TECHNOLOGY

UNIVERSITY OF CALIFORNIA

MONTANA STATE UNIVERSITY

UNIVERSITY OF NEVADA, RENO

NEW MEXICO STATE UNIVERSITY

OREGON STATE UNIVERSITY

UNIVERSITY OF OREGON

OSAKA UNIVERSITY

\author{
UNIVERSITY OF SOUTHERN CALIFORNIA \\ STANFORD UNIVERSITY \\ UNIVERSITY OF HAWAII \\ UNIVERSITY OF TOKYO \\ UNIVERSITY OF UTAH \\ WASHINGTON STATE UNIVERSITY \\ UNIVERSITY OF WASHINGTON \\ $\stackrel{*}{*} \stackrel{*}{*} \stackrel{*}{*}$ AMERICAN MATHEMATICAL SOCIETY
}

The Supporting Institutions listed above contribute to the cost of publication of this Journal, but they are not owners or publishers and have no responsibility for its content or policies.

Mathematical papers intended for publication in the Pacific Jaurnal of Mathematics should be in typed form or offset-reproduced, (not dittoed), double spaced with large margins. Please do not use built up fractions in the text of your manuscript. You may however, use them in the displayed equations. Underline Greek letters in red, German in green, and script in blue. The first paragraph or two must be capable of being used separately as a synopsis of the entire paper. Items of the bibliography should not be cited there unless absolutely necessary, in which case they must be identified by author and Journal, rather than by item number. Manuscripts, in triplicate, may be sent to any one of the editors. Please classify according to the scheme of Math. Reviews, Index to Vol. 39. All other communications should be addressed to the managing editor, or Elaine Barth, University of California, Los Angeles, California, 90024.

The Pacific Journal of Mathematics expects the author's institution to pay page charges, and reserves the right to delay publication for nonpayment of charges in case of financial emergency.

100 reprints are provided free for each article, only if page charges have been substantially paid. Additional copies may be obtained at cost in multiples of 50 .

The Pacific Journal of Mathematics is issued monthly as of January 1966. Regular subscription rate: $\$ 7200$ a year (6 Vols., 12 issues). Special rate: $\$ 36.00$ a year to individual members of supporting institutions.

Subscriptions, orders for back numbers, and changes of address should be sent to Pacific Journal of Mathematics, 103 Highland Boulevard, Berkeley, California, 94708.

PUBLISHED BY PACIFIC JOURNAL OF MATHEMATICS, A NON-PROFIT CORPORATION

Printed at Kokusai Bunken Insatsusha (International Academic Printing Co., Ltd.). 8-8, 3-chome, Takadanobaba, Shinjuku-ku, Tokyo 160, Japan.

Copyright (C) 1975 by Pacific Journal of Mathematics Manufactured and first issued in Japan 


\section{Pacific Journal of Mathematics}

Vol. 72, No. 2

February, 1977

George E. Andrews, Plane partitions. II. The equivalence of the

Bender-Knuth and MacMahon conjectures ................. 283

Lee Wilson Badger, An Ehrenfeucht game for the multivariable quantifiers

of Malitz and some applications ......................... 293

Wayne C. Bell, A decomposition of additive set functions ............ 305

Bruce Blackadar, Infinite tensor products of $C^{*}$-algebras ............. 313

Arne Brøndsted, The inner aperture of a convex set .............. 335

N. Burgoyne, Finite groups with Chevalley-type components........... 341

Richard Dowell Byrd, Justin Thomas Lloyd and Roberto A. Mena, On the retractability of some one-relator groups .....................

Paul Robert Chernoff, Schrödinger and Dirac operators with singular potentials and hyperbolic equations .................... 361

John J. F. Fournier, Sharpness in Young's inequality for convolution ....... 383

Stanley Phillip Franklin and Barbara V. Smith Thomas, On the metrizability

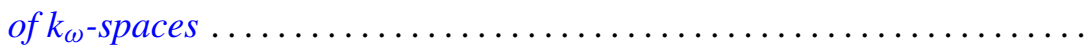

David Andrew Gay, Andrew McDaniel and William Yslas Vélez, Partially normal radical extensions of the rationals .................... 403

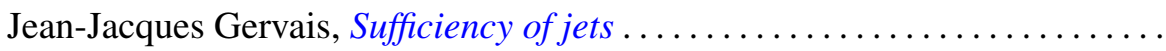

Kenneth R. Goodearl, Completions of regular rings. II . .

Sarah J. Gottlieb, Algebraic automorphisms of algebraic groups with stable maximal tori

Donald Gordon James, Invariant submodules of unimodular Hermitian forms.....

J. Kyle, $W_{\delta}(T)$ is convex.

Ernest A. Michael and Mary Ellen Rudin, A note on Eberlein compacts ...

Ernest A. Michael and Mary Ellen Rudin, Another note on Eberlein compacts ....

Thomas Bourque Muenzenberger and Raymond Earl Smithson, Fixed point theorems for acyclic and dendritic spaces.

Budh Singh Nashier and A. R. Rajwade, Determination of a unique solution of the quadratic partition for primes $p \equiv 1(\bmod 7)$.

Frederick J. Scott, New partial asymptotic stability results for nonlinear ordinary differential equations ....................

Frank Servedio, Affine open orbits, reductive isotropy groups, and dominant gradient morphisms; a theorem of Mikio Sato..........

D. Suryanarayana, On the distribution of some generalized square-full integers.................................. 